\title{
On an integral involving the logarithm function
}

\author{
Sumit Kumar Jha \\ International Institute of Information Technology \\ Hyderabad-500 032, India \\ kumarjha.sumit@research.iiit.ac.in
}

\begin{abstract}
We use the Ramanujan's master theorem to evaluate the integral

$$
\int_{0}^{\infty} \frac{x^{l-1}}{(1+x)^{m+1}} \log ^{n}(1+x) d x
$$

in terms of the digamma function, the gamma function, and the Hurwitz zeta function.
\end{abstract}

\section{Main Result}

Theorem 1. For all non-negative integers $n$ we have

$$
\begin{array}{r}
\int_{0}^{\infty} \frac{x^{l-1}}{(1+x)^{m+1}} \log ^{n}(1+x) d x=(-1)^{n} P_{n}(\psi(m+1-l)-\psi(m+1), \zeta(2, m+1)-\zeta(2, m+1-l), \\
\zeta(3, m+1)-\zeta(3, m+1-l), \cdots, \zeta(n, m+1)-\zeta(n, m+1-l)) \cdot \frac{\Gamma(m+1-l) \cdot \Gamma(l)}{\Gamma(m+1)}
\end{array}
$$

where $m$ and $l$ are complex numbers for which the integral on the left side converges, $\Gamma(s)$ is the gamma function, $\psi(s)=\frac{\Gamma^{\prime}(s)}{\Gamma(s)}$ is the digamma function, $\zeta(s, n)$ is the Hurwitz zeta function, and the polynomial $P_{n}\left(s_{1}, \cdots, s_{n}\right)$ is defined by $P_{0}=1$ and

$$
P_{n}\left(s_{1}, \cdots, s_{n}\right)=(-1)^{n} Y_{n}\left(-s_{1},-s_{2},-2 s_{3}, \cdots,-(n-1) ! s_{n}\right)
$$

where $Y_{n}$ is the familiar Bell polynomial. The first few values of the polynomials being:

$$
\begin{gathered}
P_{1}\left(s_{1}\right)=s_{1}, \\
P_{2}\left(s_{1}, s_{2}\right)=s_{1}^{2}-s_{2}, \\
P_{3}\left(s_{1}, s_{2}, s_{3}\right)=s_{1}^{3}-3 s_{1} s_{2}+2 s_{3}, \\
P_{4}\left(s_{1}, s_{2}, s_{3}, s_{4}\right)=s_{1}^{4}-6 s_{1}^{2} s_{2}+8 s_{1} s_{3}+3 s_{2}^{2}-6 s_{4} .
\end{gathered}
$$


Proof. Zave [1] proved the following series expansion:

$$
\frac{\log ^{n}(1+x)}{(1+x)^{m+1}}=(-1)^{n} \sum_{k=0}^{\infty} P_{n}\left(H_{m+k}^{(1)}-H_{m}^{(1)}, H_{m+k}^{(2)}-H_{m}^{(2)}, \cdots, H_{m+k}^{(n)}-H_{m}^{(n)}\right)\left(\begin{array}{c}
m+k \\
m
\end{array}\right)(-x)^{k}
$$

where

$$
H_{n}^{(k)}=1+\frac{1}{2^{k}}+\frac{1}{3^{k}}+\cdots+\frac{1}{n^{k}}
$$

We first recall that

$$
H_{n}^{(1)}=\psi(n+1)+\gamma
$$

$\gamma$ being the Euler's constant, and

$$
H_{n}^{(s)}=\zeta(s)-\zeta(s, n+1),
$$

for $s \geq 2$.

Now recalling the Ramanujan's master theorem [2] which states that

$$
\int_{0}^{\infty} x^{l-1}\left\{\phi(0)-x \phi(1)+x^{2} \phi(2)-\cdots\right\} d x=\frac{\pi}{\sin l \pi} \phi(-l)
$$

gives us our result whenever $m$ and $l$ are chosen such that the integral is convergent.

Substituting $n=1$ in our result Theorem 1 gives us:

\section{Corollary 2.}

$$
\int_{0}^{\infty} \frac{x^{l-1}}{(1+x)^{m+1}} \log (1+x) d x=(\psi(m+1)-\psi(m+1-l)) \cdot \frac{\Gamma(m+1-l) \cdot \Gamma(l)}{\Gamma(m+1)} .
$$

\section{References}

[1] Zave, D. A. (1976). A series expansion involving the harmonic numbers. Information Processing Letters, 5(3), 75-77.

[2] G. H. Hardy, Ramanujan. Twelve Lectures on subjects suggested by his life and work (3rd ed.), Chelsea, 1978. 